\title{
Large- and small-scale turbulent spectra in MHD and atmospheric flows
}

\author{
O. G. Chkhetiani ${ }^{1}$, A. Eidelman ${ }^{2}$, and E. Golbraikh ${ }^{3}$ \\ ${ }^{1}$ Space Research Institute, Russian Academy of Sciences, Profsoyuznaya str., 84/32 Moscow 117810, Russia \\ ${ }^{2}$ Department of Mechanical Engineering, Ben-Gurion University of the Negev, POB 653, Beer-Sheva 84105, Israel \\ ${ }^{3}$ Center for MHD Studies, Ben-Gurion University of the Negev, POB 653, Beer-Sheva 84105, Israel
}

Received: 19 July 2006 - Revised: 7 September 2006 - Accepted: 8 September 2006 - Published: 6 November 2006

\begin{abstract}
In the present review we discuss certain studies of large- and small-scale turbulent spectra in MHD and atmospheric flows performed by S. S. Moiseev and his coauthors during the last years of his life and continued by his co-authors after he passed away. It is shown that many ideas developed in these works have not lost their novelty and urgency until now, and can form the basis of future studies in this field.
\end{abstract}

\section{Introduction}

Turbulence theory has been extensively developed since Kolmogorov's works (1941) showing that major properties of turbulent flows can be described on the basis of several assumptions (Kolmogorov's hypothesis) using a certain set of universal parameters. Although there is no general theory of turbulence as yet, which remains one of the most urgent problems of the XXI century, many of its aspects have been already clarified. Professor S. S. Moiseev with his disciples and colleagues has also contributed to the study of the properties of turbulent flows. S. S. Moiseev devoted 20 years of his life to the development of so called helical turbulence theory, which plays an important part in the generation of large-scale coherent structures in turbulent media. Among the most significant results obtained by Moiseev's team is the model of large-scale vortices generation in the Earth's atmosphere (Moiseev et al., 1983a, 1983b) and, according to Ivanov et al. (1996), in the atmospheres of other planets.

According to experimental data, an interval with a universal spectrum differing from Kolmogorov's one (Eidelman et al., 2000; Branover et al., 2004) appears in scales greatly exceeding the inertial interval. It is noteworthy that the large-

Correspondence to: O. Chkhetiani

(ochkheti@mx.iki.rssi.ru) scale turbulence behavior remains insufficiently studied as yet. Therefore, the results of the study of the effect of correlation characteristics of an external random force on the spectrum generation in this spectral region obtained by Gordienko and Moiseev $(1998,1999)$ are especially significant.

In the present review, we examine the results of the study of universal spectral behavior of turbulent velocity fluctuations in MHD and atmospheric flows.

\section{Helical turbulence}

One of the results of the phenomenological approach suggested by Kolmogorov in his fundamental papers more than 60 years ago was the dependence of a two-point correlator of turbulent fluctuations of a locally homogeneous and isotropic velocity field of an incompressible fluid (and its spectral density) on the distance between these two point in the inertial interval. According to Kolmogorov, this dependence should be universal for any developed turbulent flow, where the mean turbulent energy flux from larger to smaller scales is presumed to be constant. Until today, despite a considerable progress in the study of turbulent flows, this result forms the basis of various applications of semi-empirical turbulence theories. As a matter of fact, it reflects the energy conservation law in a dynamically equilibrium system, where all turbulent energy getting into the large scales is transformed into heat in the small scales after passing all intermediate scales.

Twenty years after Kolmogorov's works, Moreau (1961) showed that the properties of mean helicity, - a scalar product of velocity by vorticity, - which is an inviscid invariant of motion, are similar to those of energy. In contrast to energy, helicity is a pseudo-scalar; it changes its sign at the inversion of coordinates. As shown by Lesieur (1991), the presence of non-zero viscosity leads to the appearance of mean helicity in a flow. Thus, helicity, along with energy, is an essential

Published by Copernicus GmbH on behalf of the European Geosciences Union and the American Geophysical Union. 
characteristic of a turbulent flow. However, the mean helicity value is often too small to affect considerably the flow behavior. One can hardly expect that a motion supported by pressure gradient only in the absence of boundaries would possess helical properties. However, as shown by many authors, in the presence of boundaries and/or external forces, helicity reaches appreciable values and can affect flow characteristics.

An important feature of helical turbulence that has first attracted the attention of researchers is the fact that it can take part in the processes of large-scale magnetic fields generation in astrophysical objects (see Vainshtein et al., 1980; Krause and Radler, 1980, and references therein). Its spectral characteristics were first studied by Brissaud et al. (1973). It has been revealed that helical turbulence possesses some exotic (in comparison with Kolmogorov's turbulence) properties due to the presence of two (instead of one) governing parameters - energy and helicity fluxes along the scales. In this paper, side by side with Kolmogorov's spectrum with $-5 / 3$ slope, helical spectrum with $-7 / 3$ slope appeared for the first time, although the authors expressed their doubts as to the existence of turbulence governed only by the helicity flux.

\subsection{Experimental results}

Experimental studies of magnetohydrodynamic (MHD) turbulence have shown that Kolmogorov's turbulence mode with the velocity spectrum slope $-5 / 3$ in the inertial range transforms into a mode with the slope close to $-7 / 3$, if the interaction parameter $N$ describing the ratio of electromagnetic to inertial forces increases. Such turbulence has been named helical, since the slope close to $-7 / 3$ can appear if its properties are determined by the helicity transfer (Branover et al., 1994). Basic properties of helical turbulence defined by the instability of large-scale motions and by the inverse energy transfer, as well as by turbulent diffusivity drop, are described in detail by Belyan et al. $(1994,1996)$ and Golbraikh et al. (1998).

Advantages of the helical model of MHD turbulence over 2-D turbulence model developed by Kraichnan (1967) and Batchelor (1969) were examined in detail by Branover et al. (1999); the main points of the issue are described below. Early experiments in MHD flows have shown that turbulence intensity of the velocity fluctuations component perpendicular to the magnetic field decreases, while their integral scales grow (e.g., Branover et al., 1965). Later on, velocity fluctuation spectra were measured, which have proved to be steeper than Kolmogorov's turbulence spectrum having the slope of $-5 / 3$. A part of them was characterized by a slope close to -3 , and the velocity fluctuations component along the magnetic field was estimated by the admixture dispersion (Kolesnikov and Tsinober, 1974). The decrease in the dispersion scale in the magnetic field direction was interpreted as a decrease in this velocity component. These data made up the reasoning in favor of the application of 2-D turbulence model of MHD turbulence (Kit and Tsinober, 1971).

However, further experiments have shown that the velocity fluctuations field is essentially three-dimensional (Alemany et al., 1979; Seluto, 1984). Slopes of the velocity spectra steeper than $-5 / 3$ were obtained in MHD flows (Branover et al., 1994), where modes with $-11 / 3$ and $-7 / 3$ slopes were revealed, and the latter was named the helical turbulence mode. The analysis of the velocity spectra obtained in MHD flows (Kolesnikov and Tsinober, 1974; Alemany et al., 1979; Sommeria, 1983; Sukoriansky and Branover, 1988; Platnieks and Seluto, 1989) has shown that -3 slope is observed, with rare exceptions, near the grids. The spectral slope downstream of the grids is usually close to $-7 / 3$, if the interaction parameter $N$ is in the range of $0.2<N<1.4$, and even steeper, $-11 / 3$, - at $2<N<15$. Here $N=\sigma B^{2} L / \rho U$, where $\sigma$ and $\rho$ are the fluid electrical conductivity and density, respectively, $B$ is the flux density of the magnetic field, $U$ and $L$ - characteristic velocity and scale of the flow.

Thus, MHD turbulence properties, such as a decrease of energy dissipation, growth of velocity fluctuation scales, anisotropy of the three components of velocity fluctuations, spectral slope equal to $-7 / 3$, are in good agreement with the properties of helical turbulence (see Branover et al., 1999). Helicity generation in MHD flows, which is the cause of helical MHD turbulence, was revealed by Chkhetiani et al. (1998). An essentially 3D character of the helical turbulence model corresponds to actual motions, which remain three-dimensional even under the conditions of a strong constraint. Thus, we suppose that the application of the helical turbulence model to the description of intrinsic properties of real turbulent flows is quite adequate.

To reveal the helical turbulence mode in different laboratory and geophysical conditions and to establish its quantitative characteristics, velocity spectra were examined. The analysis of experiments demonstrates that the phenomenon of helical turbulence exists in turbulent flows in various conditions. The spectral slope $-7 / 3$ characteristic of MHD helical turbulence was revealed in the laboratory studies of flows with convection, rotation, stratification, as well as in numerical modeling of such flows.

Velocity spectra with the slopes -2.3 and -2.46 were obtained for turbulence generated by an unstable density front in stratified fluid experiments under rotation (Griffiths and Hopfinger, 1984). Similar results were obtained in a rotationally dominated turbulent flow, where the spectral slope was evaluated as -2.5 (Mory and Hopfinger, 1986). The experiments in stably stratified turbulence have revealed spectra with a slope "...slightly greater than -2 " (Istweire and Helland, 1985). The authors note that the observed inverse energy cascade corresponds to the predictions for twodimensional turbulence, but the velocity spectra do not show any evidence that the collapsed turbulence has become purely two-dimensional. In this experiment, the velocity spectrum was obtained in a flow of salt-stratified water streamdown of 
Table 1. PArameters of atmospheric helical turbulence spectra (ABL - boundary layer, TR - troposphere, STR - low stratosphere).

\begin{tabular}{lcccl}
\hline Investigators & $\varepsilon, 10^{-4} \mathrm{~m}^{2} / \mathrm{s}^{3}$ & $\eta, 10^{-8} \mathrm{~m} / \mathrm{s}^{3}$ & $L_{\eta}, 10^{3} \mathrm{~m}$ & Notes \\
\hline Vinnichenko and Dutton, 1969 & 4 & 10 & 4 & Flights, TR \\
Lenschow, 1970 & 42 & 2100 & 0.2 & Flights, strong ABL convection \\
Caughey and Palmer, 1979 & 0.5 & 20 & 0.25 & Tower, over ABL, type 1 \\
& 12.3 & 180 & 0.68 & Tower, ABL, type 1 \\
Mason, King, 1985 & 280 & 2800 & 0.001 & Near a surface, strong wind \\
Lilly and Petersen, 1983 & 0.9 & 0.1 & 90 & Flights, TR, type 1 \\
Nastrom et al., 1984 & 0.75 & 0.05 & 150 & GASP flights, type 1 \\
Nastrom et al., 1987 & & & GASP flights, strong wind, type 2 \\
& 2.6 & 1.2 & 21.7 & over the mountains, TR \\
& 3.0 & 2.3 & 13 & over the mountains, STR \\
& 0.44 & 0.41 & 10.7 & over the plain, STR \\
& 1 & & GASP flights, slight wind, type 2 \\
& 2 & 0.77 & 13 & over the mountains, TR \\
& 0.32 & 1.35 & 14.8 & over the mountains, STR \\
& 0.67 & 0.27 & 11.8 & over the plain, TR \\
& 0.26 & 0.74 & 9 & over the plain, STR \\
& & 13 & over the ocean, STR \\
& 36 & 300 & 1.2 & Balloon zond at 0.5-16 km \\
Fritts and Chou, 1987 & 1.15 & 40 & 0.29 & slight wind \\
Eazarev et al., 1994 & 0.95 & 5.6 & 1.7 & Radar vert. meas. at 9-18 km \\
& 0.29 & 0.87 & 3.3 & Radiosondes up to13 km, type 1 \\
\hline & & &
\end{tabular}

an array of vertical rods. We estimate the spectral slope in the section $x / M=60(M=3.81 \mathrm{~cm})$ as rather close to $-7 / 3$ within the range of scales covering a decade. Besides, in the adjacent range covering a decade in larger scales, the spectral slope is close to $-5 / 3$.

Our further studies have shown that spectra with the slope close to $-7 / 3$ were also obtained in the atmosphere and in the ocean (Monin and Ozmidov, 1985). Twenty six tapes of wind measurements obtained during long-distance flights were studied by Lilly and Petersen (1983), and the corresponding wind spectral slope was about -2.2 . A detailed study of these spectra revealed two ranges separated by the scale of $90 \mathrm{~km}$ with slopes $-7 / 3$ in larger scales and $-5 / 3$ in smaller ones. The slope of 130 stratospheric wind spectra obtained during Global Atmospheric Sampling Program (GASP) was estimated as -2.2 and -2.5 for the synoptic scale range (Nastrom and Gage, 1983), which is close to $-7 / 3$. Our estimation of GASP spectra obtained in 1670 flights at the latitudes from $30^{\circ} \mathrm{S}$ to $60^{\circ} \mathrm{N}$ (Nastrom and Gage, 1985) gives a slope close to $-7 / 3$ for the meridional wind component in the range of synoptic scales exceeding $300 \mathrm{~km}$ (Branover et al., 1999). Here the adjacent mesoscale range can be satisfactorily approximated by $-5 / 3$ slope. Wind spectra characterizing in more detail the mesoscale range of GASP data are given by Nastrom et al. (1987) for three different regions - over plains, mountains and ocean. The authors note that steepened spectral slopes ( $-7 / 3$ according to our estimation) correspond to wind velocity fluctuations with scales below $15-23 \mathrm{~km}$.

Table 1 summarizes the main properties of atmospheric helical turbulence spectra. Spectra include adjacent ranges with slopes close to $-5 / 3$ and $-7 / 3$ and a characteristic scale $L_{\eta}=\varepsilon / \eta$ of their intersection. As observations show, under atmospheric conditions there exist several $L_{\eta}$ scales. They characterize the situation of several energy and helicity inputs generating a complex wind spectrum, where the spectral slope transforms with the growing wavenumber from $-7 / 3$ to $-5 / 3$ (type 1 ) and from $-5 / 3$ to $-7 / 3$ (type 2 ). If the range scale is limited, as in laboratory flows or in the atmospheric boundary layer, only one scale or even one range is observed. Energy $\varepsilon$ and helicity $\eta$ transfer rates $\left(\eta=\varepsilon / L_{\eta}\right)$ estimated on the basis of the velocity spectra in flows of various scales are presented in Table 1.

We have independently estimated $\eta$ taking into account the Earth rotation as

$\eta=2 t^{-1} w \Omega \sin \theta$,

where $t$ is the characteristic time of the spectral maximum, $w$ is the vertical wind velocity; $\Omega$ is the angular velocity of the Earth rotation $\left(\Omega=7.27 \times 10^{-5} \mathrm{rad} / \mathrm{s}\right)$; and $\theta$ is the latitude. The helicity transfer rate in synoptic scales with the characteristic time $t=100 \mathrm{~h}$, vertical velocity $u=3 \mathrm{~m} / \mathrm{s}$ at the latitude $\theta=45^{\circ}$ is $\eta=0.086 \times 10^{-8} \mathrm{~m} / \mathrm{s}^{3}$. The helicity transfer rate in the atmospheric boundary layer scales, where 
$t=200 \mathrm{~s}$, and $w, \Omega, \theta$ are the same as in the previous case, is $150 \times 10^{-8} \mathrm{~m} / \mathrm{s}^{3}$. These results are close to the values obtained from measured spectra using the energy transfer rate and the scale $L_{\eta}$ (see Lilly and Petersen, 1983; Nastrom et al., 1984; Caughey and Palmer, 1979 in Table 1).

\subsection{Helical Spectrum}

As already noted above, spectral characteristics of the helical turbulence were first studied by Brissaud et al. (1973). The authors studied for the first time the invariant spectral behavior of the helicity flux $\eta$, side by side with that of the energy flux $\varepsilon$. Here the helical spectrum $-7 / 3\left(E(k) \sim \eta^{2 / 3} \boldsymbol{k}^{-7 / 3}\right)$ appears along with the Kolmogorov's spectrum $-5 / 3$. However, according to the approach based on the effective correlation time suggested by Kraichnan, the arising $-7 / 3$ spectrum characterizes the absence of energy flux $(\varepsilon=0)$. Thus, in the present limiting case, the external turbulence source had to generate a helicity flux in the absence of an energy flux, which is rather problematic from the physical standpoint. However, as follows from the experimental data examined in the previous section, the helical spectrum is not at all exotic, being observed in various flows. However, in these flows the energy flux is also nonzero. Thus, a certain discrepancy between the model and the experiments arises.

Another attempt to analyze the spectral behavior of helical turbulence was made by Moiseev and Chkhetiani (1996). Having investigated symmetry characteristics of the NavierStokes equation written for a characteristic functional, the authors obtained similar spectra (Brissaud et al., 1973) without making, however, any suggestions regarding the magnitude of the energy flux. Thus, they have proved the physical character of the helical spectrum with the slope $-7 / 3$. On the other hand, the authors have obtained a subdivision of the inertial interval into two intervals separated by a characteristic scale $L_{\eta}=\varepsilon / \eta$. Kolmogorov's spectrum is generated in scales greatly exceeding the latter, whereas the helical spectrum - in much smaller scales. This can explain convex bends arising in turbulent spectra. However, besides such convex bends, concave ones are also observed in turbulent spectra. This theory does not explain the appearance of such bends.

Apparently, there are some other factors, besides the energy and helicity fluxes, affecting the behavior of the spectral density in the inertial interval. In order to reveal such factors, Golbraikh and Moiseev (2002) have studied the asymptotic behavior of the structure function $D(r)$ of the turbulent velocity field in the case where $\varepsilon$ and $\eta$ are governing parameters in the inertial and dissipative intervals, and the kinematic viscosity is an additional governing parameter in the dissipative interval. As follows from the paper, the spectral function behavior in the inertial interval depends on the behavior of the governing parameters in the dissipative interval. Kolmogorov's spectrum arises in the inertial interval close to the dissipative interval, when the helicity flux in the latter stops being a governing parameter and depends on the energy flux.
The helical spectrum, however, characterizes the case where the helicity flux determines the energy flux in the dissipative interval.

The results of the present paper have shown that the properties of the structure function in the inertial interval are affected by the behavior of the determining parameters in adjacent intervals. Hence, the large-scale behavior of turbulence should also affect its characteristics in the inertial interval.

\section{Large scale spectra}

\subsection{Universal spectrum}

The universality of the behavior of the turbulent velocity field spectral density in the region of small wave numbers was emphasized in papers dealing with the study of MHD flows in square channels placed in a transverse uniform magnetic field (see Branover et al. (1999) and references therein). It does not follow from Kolmogorov's theory that the dependence of spectral energy density on the wave vector $\boldsymbol{k}$ obtained in his works is valid for all scales. In fact, when considering various scale intervals, it is assumed that stationary incompressible homogeneous turbulence at high Reynolds numbers is subdivided into intervals, each of them described by its own law.

The studies of large-scale turbulence were less successful, although it was known that large-scale structures often play a very important role in turbulent flows. The generation of large scales due to the laminar flow stability loss is a fundamental property of turbulence origination suggested by Landau (1944). The universality of large scales formed due to the inverse energy cascade in two-dimensional turbulence has been known for a long time and studied both experimentally and numerically (see, for example, Lesieur, 1991; Kraichnan and Montgomery, 1980, and references therein).

Velocity spectra obtained in MHD experiments at various values of the interaction parameter $N$ are shown in Fig. 1 . Spectral density $E(k)$ is shown in a dimensionless form $\boldsymbol{k} E(k) / u^{2}$, where $u^{2}$ is the kinetic energy of velocity fluctuations. The spectral slope in frequency-weighed coordinates has an index exceeding that of the spectral density representation by a unity. In the large-scale range covering almost a decade, these spectra can be well approximated by a straight line with the slope of $4 / 3$ corresponding to the spectral slope 1/3. MHD turbulence spectrum A shown in Fig. 1 has $-7 / 3$ slope in the small-scale range. With increasing interaction parameter and turbulence transition into an intermittent mode, the slope becomes close to -4 for the spectrum C. However, despite this, the spectral slope in the largescale range remains close to $1 / 3$.

The $1 / 3$ spectrum is also observed in a stable atmospheric boundary layer (Caughey, 1977) and in such excited dynamic systems as cirrus clouds (Gultepe and Starr, 1995), where the spectral slope in the small-scale range is also close to $-7 / 3$. 


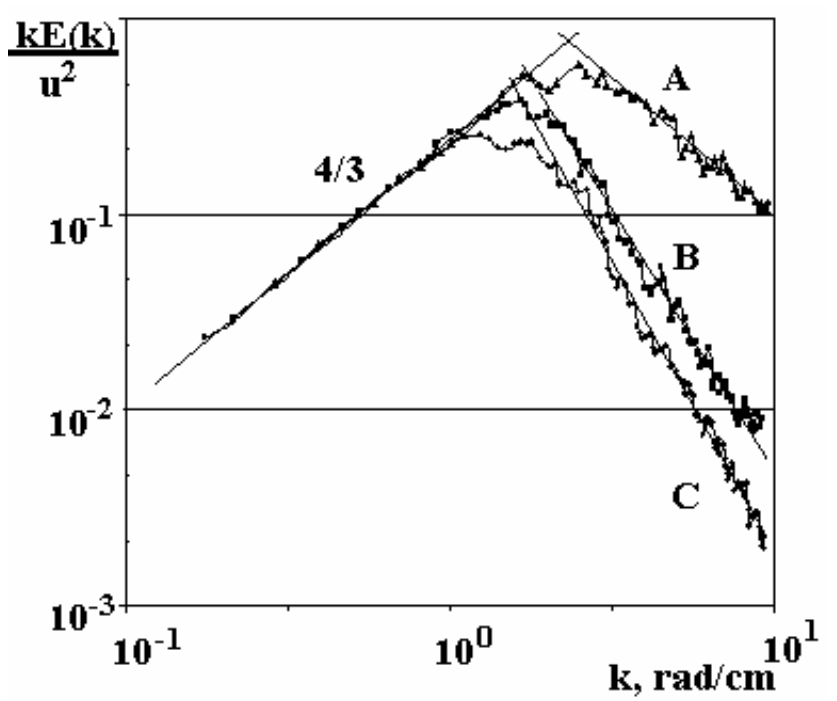

Fig. 1. Dimensionless velocity spectra measured along the axis of a turbulent channel flow with a universal large-scale range. Smallscale modes vary with the interaction parameter: $A-N=0.67$; $B$ - 1.57; $\mathrm{C}-15.6$. The spectrum $\mathrm{A}$ is shifted by half an order upwards along the line with the slope $4 / 3$ corresponding to $1 / 3$ for the spectral density (according to Branover et al., 2004).

There are also turbulence spectra where the large-scale 1/3 spectrum coexists with Kolmogorov's spectrum $-5 / 3$ in the small-scale range. Examples of such velocity spectra observed in the atmospheric boundary layer are shown in Fig. 2. Here the spectral slope in frequency-weighed coordinates has an index exceeding that of spectral density representation by a unity, as in Fig. 1. Spectra with the slope of $1 / 3$ in the largescale range were obtained both in laboratory and atmospheric turbulent flows with different slopes in small scales.

The main determining factors affecting turbulence behavior over large scales $L_{f}\left(L_{f} \gg l_{i}\right.$, where $l_{i}$ is the characteristic scale of the inertial interval) are an external force and the integral energy introduced into the turbulence by this force. This is a region with scales greatly exceeding the characteristic scale of the external force or the external turbulence scale $L_{0}$. According to Branover et al. (2004), a governing parameter in this region is the parameter

$\xi=\int \frac{d U_{0}^{\prime 2}}{d t} d V=f_{0}^{2} r_{0}^{3} \tau_{0}$,

where $U_{0}^{\prime}$ are turbulent fluctuations generated by the external force, and $f_{0}, r_{0}$ and $\tau_{0}$ are characteristic values of the amplitude, scale and correlation time of the random external force (Gordienko and Moiseev, 1998, 1999).

Following the dimensional theory in this region, the spectral density

$E(k) \sim \xi^{2 / 3} k^{1 / 3}$,

since the dimension of $\xi$ is $\mathrm{m}^{5} / \mathrm{s}^{3}$.

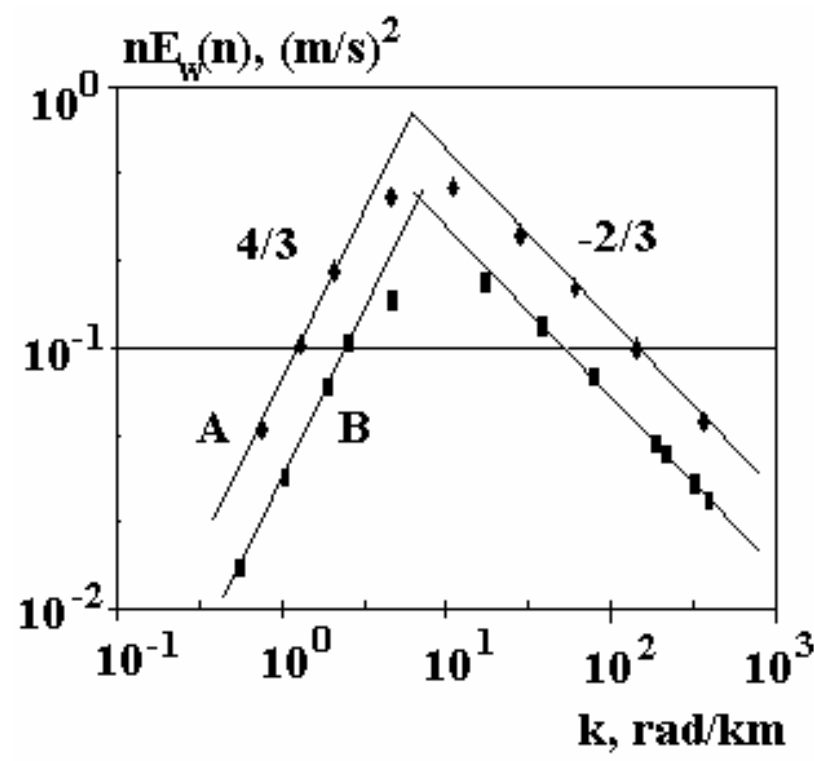

Fig. 2. Spectrum of vertical wind components sampled crosswind from an aircraft: A - flight 11, B - flight 13. Straight lines with $4 / 3$ and $-2 / 3$ slopes drawn by us correspond to $1 / 3$ and $-5 / 3$ slopes, respectively, for the spectral density (adapted from Caughey, 1977).

It is noteworthy that the large-scale turbulence is described only by force parameters, and not by small-scale turbulence properties. Thus, there exist both experimental and theoretical prerequisites for the universal behavior of the developed turbulence in large scales. Apparently, formal difficulties in the validation of Kolmogorov's spectrum connected with infrared divergence were concealing a new physical phenomenon, namely, the universality of large-scale turbulence.

\subsection{Connection with the inertial interval}

As noted above, asymptotic connections between different intervals of the spectrum lead to its modification. On the one hand, the range of energy generation by external sources, their scales, topological properties and other features affect the behavior of the adjacent region of the inertial interval (Golbraikh and Eidelman, 2006). On the other hand, the dissipation range affects the remaining region of the inertial interval (Golbraikh and Moiseev, 2002).

We assume that within the scope of the external force, where $L \approx r_{0}$, the governing parameters are $\xi$, as well as $\varepsilon$ and $\eta$ - energy and helicity fluxes over the spectrum. Under the condition that at $r \gg r_{0}$ the structure function should be independent of $\varepsilon$ and $\eta$ and turn into $D(r) \sim \xi^{2 / 3} r^{-4 / 3}$, whereas in the inertial interval at $r \ll r_{0}$ the dependence of $D(r)$ on the parameter $\xi$ should vanish, we obtain that in the inertial interval (Golbraikh and Eidelman, 2006)

$D(r)=C_{\delta_{L}}\left(\frac{\varepsilon^{2}}{\eta}\right)^{2 / 3}\left(\frac{\eta}{\varepsilon}\right)^{\delta_{L}} r^{\delta_{L}}$

where $C_{\delta_{L}}$ is a certain constant. 


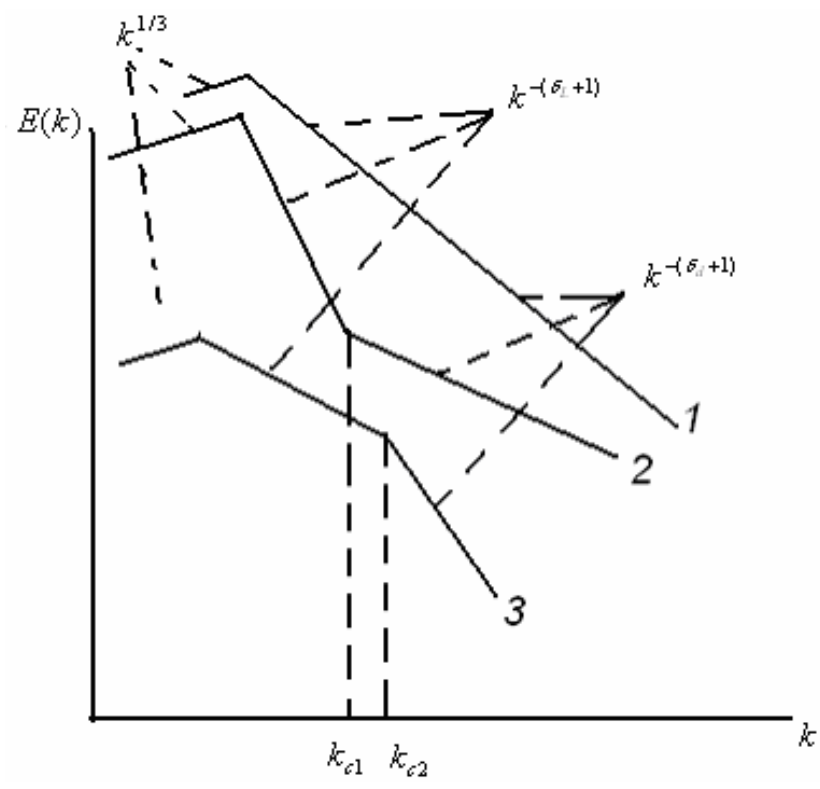

Fig. 3. Possible types of spectral function $E(k)$ :

$1-$ at $\delta_{d}=\delta_{L}$;

$2-$ at $\delta_{L}>\delta_{d}$;

$3-$ at $\delta_{L}<\delta_{d}$.

The form of this expression coincides with that of (11) derived by Golbraikh and Moiseev (2002).

$D(r)=C_{\delta_{d}}\left(\frac{\varepsilon^{2}}{\eta}\right)^{2 / 3}\left(\frac{\eta}{\varepsilon}\right)^{\delta_{d}} r^{\delta_{d}}$

where $C_{\delta_{d}}$ is a certain constant. However, in the work of Golbraikh and Moiseev (2002), it is a consequence of the asymptotic behavior of the correlation function at the transition from the dissipative interval to the inertial one, and the value $\delta_{d}$ is determined by the behavior of $\varepsilon$ and $\eta$ in the dissipative interval. Meanwhile, $\delta_{L}$ is determined by the behavior of these magnitudes in large scales.

Figure 3 qualitatively shows various dependencies of the spectral density $E(k)$ corresponding to Eqs. (3) and (4). As Fig. 3 (curve 1) shows, in the case of $\delta_{d}=\delta_{L}$, the function $E(k)$ has no bends. In the opposite case, a bend appears in the inertial interval within the scale

$r_{c}=l_{c}\left(\frac{C_{\delta_{d}}}{C_{\delta_{L}}}\right)^{\delta_{L}-\delta_{d}}$

where $C_{\delta_{d}}$ is a constant appearing in a similar expression for $D(r)\left(\right.$ Eq. 4), and $l_{c}=\frac{\varepsilon}{\eta}$ is the scale of spectra crossing at equal constants in Eq. (5). In Fig. 3, $k_{c}=\frac{1}{r_{c}}$ and the lines 2 and 3 correspond to the cases $\delta_{L}>\delta_{d}$ and $\delta_{L}<\delta_{d}$, respectively, whereas in the scales $\tilde{L} \gg r_{0}$, the spectrum remains universal $\left(E(k)=C_{\xi} \xi^{2 / 3} k^{1 / 3}\right)$.

More complicated spectra are shown in Fig. 4. These spectra were obtained by Mason and King (1985) when studying the overflow of a hill in the atmospheric boundary layer.

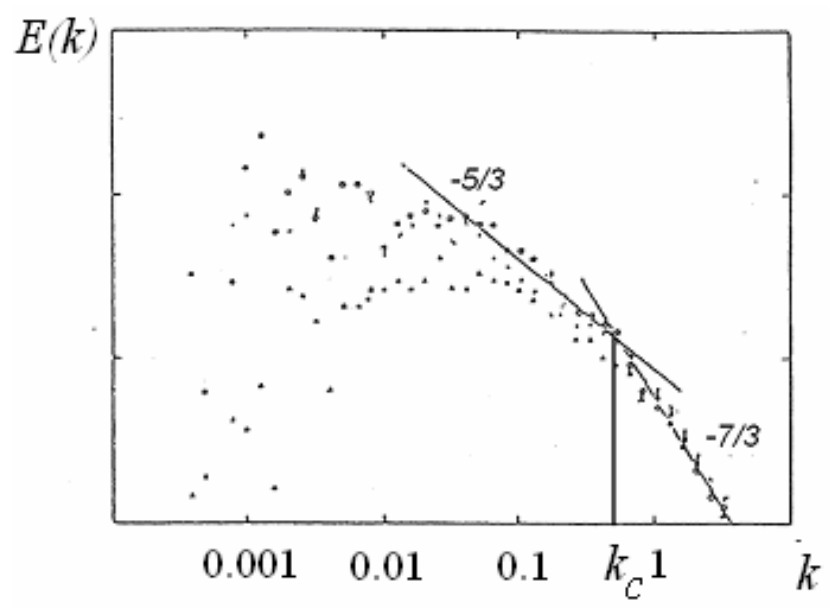

Fig. 4. Turbulence power spectra obtained by Mason and King at the altitudes $\sim 14 \mathrm{~m}$ in the atmospheric boundary layer (according to Mason and King, 1985).

These spectral dependencies refer to the case of $\delta_{L}<\delta_{d}$ and a convex bend. Here, as follows from the data, $r_{c} \approx 2-3 \mathrm{~m}$, and the external scale is close to $80 \mathrm{~m}$. These spectra allow us to make certain conclusions about the structure of turbulence under study. An external source connected with a hill overflow generates a large-scale field of turbulent fluctuations in the external scale region, whose properties are determined by the energy flux $\varepsilon$ along the spectrum and its power $\xi$. In this case, the helicity flux $\eta$ depends on these values, which results in Kolmogorov's spectrum in the long-wave region of the inertial interval. On the other hand, in the dissipative interval the situation is different, and the helicity flux, side by side with the viscosity (Golbraikh and Moiseev, 2002), determine the properties of the energy flux, which generates a helical spectrum (with the slope -7/3) in the short-wave region of the inertial interval.

Thus, the formation of complicated spectra of developed turbulence in the inertial interval is connected with the fact that its different parts are affected by different processes. An external turbulence source affects its large-scale behavior, whereas the dissipation affects the small-scale behavior.

\section{Conclusions}

In conclusion, we would like to emphasize that in the studies performed in cooperation with Prof. Moiseev or under his supervision, mean helicity, side by side with energy, plays a considerable part in the behavior of turbulent flows. The helicity impact on the flow characteristics depends on its magnitude. The presented data show that velocity spectra of real turbulent MHD flows and turbulent flows with rotation or stratification in the atmosphere are close to model spectra of helical turbulence with the slopes of $-7 / 3$ and $-5 / 3$ in various scales. The properties of the spectra in the inertial 
interval are affected by the behavior of the determining parameters in adjacent intervals. Particularly, the dissipation interval affects the inertial range in small scales, and the universal interval of large scales affects the inertial range in large scales. Further development of the helical turbulence model is necessary not only for the development of turbulence theory. These results are of great practical importance. Thus, the model of large-scale vortical structures generation suggested in early $80 \mathrm{~s}$ by Prof. Moiseev with co-authors is based on a considerable effect of helicity on their generation. It was confirmed in unique full-scale experiments aimed at the study of typhoons origination (Lazarev et al., 1990, 1992; Lazarev and Moiseev, 1992) in the tropical atmosphere. Unfortunately, at present such complex studies of the atmosphere properties during typhoons origination are not under way. However, we hope for the revival of interest in such studies because of ever growing damages caused by typhoons (or hurricanes) in many countries.

The authors of the present brief review were lucky to work and communicate with Prof. Semen Moiseev for many years. The character of this truly great scientist, his love of physics, original way of thinking and inexhaustible scientific curiosity have survived in his works and in our memory.

Acknowledgements. O. C. is grateful to V. M. Ponomarev and M. V. Kurgansky for fruitful discussion. The work was supported in part by the Russian Foundation for Basic Research, project nos. 05-05-64735 and 05-05-90596 (RFBR-NSC).

Edited by: N. S. Erokhin

Reviewed by: three referees

\section{References}

Alemany, A., Moreau, R., Sulem, P. L., and Frisch, U.: Influence of an external magnetic field on homogeneous MHD turbulence, J. de Mecanique, 18, 277-313, 1979.

Batchelor, G. K.: Computation of the energy spectrum in homogeneous two-dimensional turbulence, Phys. Fluids, 12, 233-239, 1969.

Belyan, A. V., Moiseev, S. S., and Chkhetiani, O. G.: On eddy viscosity in helical turbulence, Physics - Doklady, 39, 13-15, 1994.

Belyan, A. V., Golbraikh, E. I., Moiseev, S. S., and Chkhetiani, O. G.: Instability of second moments in helical turbulence, Tech. Phys. Lett., 22, 187-188, 1996.

Branover, H. H., Slyusarev, N. M., and Shcherbinin, E. V.: Some results of measuring turbulent velocity pulsations in mercury flow under transverse magnetic field, Magnitnaya Gidrodinamika, 1, 33-36, 1965.

Branover, H., Eidelman, A., Nagorny, M., and Kireev, M.: MHD simulation of quasi-two-dimensional geophysical turbulence, in: Advances in Turbulent Studies, edited by: Branover, H. and Unger, Y., AIAA, Washington, USA, 64-79, 1994.

Branover, H., Eidelman, A., Golbraikh, E., and Moiseev, S.: Turbulence and Structures, Academic Press, N.Y., 1999.
Branover, H., Eidelman A., Golbraikh, E., Gordienko, S., and Moiseev, S.: On the universality of large-scale turbulence, Phys. Fluids, 16, 845-847, 2004.

Brissaud, A., Frisch, U., Leorat, J., Lesieur, M., and Mazure, A.: Helicity cascades in fully developed isotropic turbulence, Phys. Fluids, 16, 1366-1367, 1973.

Caughey, S. J.: Boundary-layer turbulence spectra in stable conditions, Boundary-Layer Met., 11, 3, 3-14, 1977.

Caughey, S. J. and Palmer, S. G.: Some aspects of turbulence structure through the depth of the convective boundary layer, Quart. J. Roy. Meteorol. Soc., 105, 811-827, 1979.

Chkhetiani, O., Moiseev, S., and Golbraikh, E.: Helicity generation in turbulent MHD flows, Zh. Eksp. Teor. Fiz., 114, 946-955, 1998.

Eidelman, A., Branover, H., Moiseev, S. S., and Gordienko, S. N.: Large-scale turbulence universality and the study of extreme weather events, Phys. Chem. Earth (B), 25, 35-38, 2000.

Endlich, R. M., Singleton, R. C., and Kaufman, J. W.: Spectral analysis of detailed vertical wind speed profiles, J. Atmosph. Sci., 26, 1030-1041, 1969.

Fritts, D. C. and Hua-Guo Chou: An investigation of the vertical wavenumber and frequency spectra of gravity wave motions in the lower stratosphere, J. Atmos. Sci., 44, 3610-3624, 1987.

Golbraikh, E., Chkhetiani, O., and Moiseev, S.: The role of helicity in turbulent MHD flows, J. Exp. Theor. Phys., 87, 95-100, 1998.

Golbraikh, E. and Moiseev, S.: Different spectra formation in the presence of helical transfer, Phys. Lett. A, 305, 173-175, 2002.

Golbraikh, E. and Eidelman, A.: On the structure of complicated turbulent spectra, Physica A, accepted, 2006.

Gordienko, S. N. and Moiseev, S. S.: Parametrization and universal properties of turbulence of an incompressible fluid, J. Exp. Theor. Phys. Lett., 68, 204-209, 1998.

Gordienko, S. N. and Moiseev, S. S.: Structure of turbulent flows of incompressible fluids and the parametrization of turbulence, $\mathrm{Zh}$. Eksp. Teor. Fiz., 116, 1630-1647, 1999.

Griffiths, R. W. and Hopfinger, E. J.: The structure of mesoscale turbulence and horizontal spreading at ocean fronts, Deep-Sea Res., 31, 245-269, 1984.

Gultepe I. and Starr, D.: Dynamical structure and turbulence in cirrus clouds: Aircraft observations during FIRE, J. Atmosph. Sci., 52, 4159-4182, 1995.

Itsweire, E. C. and Helland, K. N.: Turbulent mixing and energy transfer in stably stratified turbulence, 7 th Symp. on Turbulence and Diffusion. November 12-15, 1985, Boulder, Colorado, 172$175,1985$.

Ivanov, M. F., Gal'burt, V. A., and Fortov, V. E.: On a possible mechanism of the formation of large-scale disturbances in Jupiter's atmosphere as a result of the falling of fragments of Comet Schoemaker-Levy 9, J. Exp. Theor. Phys. Lett., 63, 813817, 1996.

Kit, E. G. and Tsinober, A. B.. Possibility of creating and investigating two-dimensional turbulence in a strong magnetic field, Translated from Magnitnaya Gidrodinamika, 3, 27-34, 1971.

Kolesnikov, Yu. B. and Tsinober, A. B.: Experimental investigation of two-dimensional turbulence behind a grid, Dokl. Akad. Nauk, Mekh. Zhidk. Gaza, 4, 146-150, 1974.

Kolmogorov, A. N.: Local structure of turbulence in an incompressible fluid at very high Reynolds numbers, Dokl. AN SSSR, 30, 299-303, 1941. 
Kraichnan, R. H.: Inertial ranges in two-dimensional turbulence, Phys. Fluids, 10, 1417-1423, 1967.

Kraichnan, R. H. and Montgomery, D.: Two-dimensional turbulence, Reports Orog. Phys., 43, 547-619, 1980.

Krause, F. and Radler, K.-H.: Mean-Field Magnetohydrodynamics and Dynamo Theory, Pergamon Press, Oxford, 1980.

Landau, L. D.: On the problem of turbulence, DAN SSSR, 44, 339342, 1944.

Lazarev, A. A., Mazurov, A. N., Moiseev, S. S., Pokrovskaya, I. V., Rutkevitch, P. B., Sagdeev, R. Z., Skatchkov, V. A., Smirnov, N. K., Tunegolovetc, V. P., Tur, A. V.,and Chernikov, I. V.: Experimental studies of large-scale structure origination in tropical atmosphere (expedition Typhoon-89), in: Nonlinear Dynamics of Structures, Proc. Intern. Symp. on Generation of LargeScale Structures in Continuous Media, 11-20 June 1990, PermMoscow, USSR, 327-336, 1990.

Lazarev, A. A., Moiseev, S. S., and Zimin, V. D.: Large-scale processes in tropical atmosphere: Mathematical model, Space Research Institute, RAS, Pr-1840, 27, 1-27, 1992.

Lazarev, A. A. and Moiseev, S. S.: Geophysical precursors of early stages of cyclogenesis, Space Research Institute, RAS, Pr-1844, $31 \mathrm{pp}, 1992$.

Lazarev, A., Schertzer, D., Lovejoy, S., and Chigirinskaya, Y.: Unified multifractal atmospheric dynamics tested in the tropics: Part II: Vertical scaling and generalized scale invariance, Nonlin. Processes Geophys., 1, 115-123, 1994, http://www.nonlin-processes-geophys.net/1/115/1994/.

Lenschow, D. H.: Airplane measurements of planetary boundary layer structure. J. Appl. Meteorol., 9, 874-884, 1970.

Lesieur, M.: Turbulence in Fluids,. Kluwer Acad. Publ., 1991.

Lilly, D. K. and Petersen, E. L.: Aircraft measurements of atmospheric kinetic energy spectra, Tellus, 35A, 379-382, 1983.

Mason, P. J. and King, J. C.: Measurements and predictions of flow and turbulence over an isolated hill of moderate slope, Quart. J. R. Met. Soc., 111, 617-640, 1985.

Moiseev, S. S., Sagdeev, R. Z., Tur, A. V., Khomenko, G. A., and Shukurov, A. M.: Physical mechanism of amplification of vortex disturbances in atmosphere, Soviet Phys. Dokl., 28, 926-928, 1983a.

Moiseev, S. S., Sagdeev, R. Z., Tur, A. V., Khomenko, G. A., and Yanovskii, V. V.: Theory of the origin of large-scale structures in hydrodynamic turbulence, Sov. Phys., JETP, 58, 1149-1153, 1983b.
Moiseev, S. S. and Chkhetiani, O. G.: Helical scaling in turbulence, J. Exp. Theor. Phys., 83, 192-198, 1996.

Monin, A. S. and Ozmidov, R. V.: Turbulence in the Ocean, D. Reidel, Dordrecht, Holland, 1985.

Moreau, J. J.: Constants d'un ilot tourbillonnaire en fluide parfait barotrope, C. R. Acad. Sci. Paris, 252, 2810-2812, 1961.

Mory, M. and Hopfinger, E. J.: Structure functions in a rotationally dominated turbulent flow, Phys. Fluids, 29, 2140-2146, 1986.

Nastrom, G. D. and Gage, K. S.: A first look at wavenumber spectra from GASP data, Tellus, 35A, 383-388, 1983.

Nastrom, G. D., Gage, K. S., and Jasperson, W. H.: Kinetic energy spectrum of large- and mesoscale atmospheric processes, Nature, 310, 36-38, 1984.

Nastrom, G. D. and Gage, K. S.: A climatology of atmospheric wavenumber spectra of wind and temperature observed by commercial aircraft, J. Atmos. Sci., 42, 950-960, 1985.

Nastrom, G. D., Fritts, D. C., and Gage, K. S.: An investigation of terrain effects on the mesoscale spectrum of atmospheric motions, J. Atmos. Sci., 44, 3087-3096, 1987.

Platnieks, I. and Seluto, S. F.: The effect of initial and boundary conditions upon the formation and development of MHD turbulence structure, edited by: Lielpeteris, J. and Moreau, R., Kluwer Acad. Publ., 433-439, 1989.

Seluto, S. F.: Magnetic field effect on the formation of turbulence structure behind grids of various configurations, Magnitnaya Gidrodinamika, 3, 55-60, 1984.

Sommeria, J.: Two-dimensional behaviour of MHD fully developed turbulence, J. de Mecan. Theor. Appl., Numero special, 169-190, 1983.

Sukoriansky, S. and Branover, H.: Turbulence peculiarities caused by interference of magnetic field with the energy transfer phenomena, in: Current Trends in Turbulence Research, edited by: Branover, H., Mond, M., and Unger, Y., Proc. 5th Beer-Sheva Int. Seminar on MHD Flow and Turbulence, Beer-Sheva, Israel, 2-6 March 1987, 87-99, 1988.

Vainshtein, S. I., Zel'dovich, Ya .B., and Ruzmaykin, A. A.: Turbulent Dynamo in Astrophysics, Nauka, Moscow, 1980.

Vinnichenko, N. K. and Dutton, J. A.: Empirical studies of atmospheric structure and spectra in the free atmosphere, Radio Sci., 4, 1115-1126, 1969. 